(C) <2019>. This manuscript version is made available under the CC-BY-NC-ND 4.0 license http://creativecommons.org/licenses/by-nc-nd/4.0/

The definitive publisher version is available online at 10.1016/j.ienj.2019.01.003 
TITLE: SUICIDE IN OLDER PEOPLE, ATTITUDES AND KNOWLEDGE OF EMERGENCY NURSES: A

\section{MULTI-CENTRE STUDY}

\section{Author names and affiliations:}

${ }^{1,2^{*}}$ Margaret Fry RN, PhD, ${ }^{1}$ Kirsten Abrahamse, RN, ${ }^{1}$ Steve Kay RN, MN and ${ }^{1,2}$ Rosalind $\mathrm{M}$ Elliott, RN PhD

${ }^{1}$ Northern Sydney Local Health District

St Leonards NSW 2065

Australia

${ }^{2}$ Faculty of Health

University of Technology Sydney

PO Box 123

Broadway NSW 2007

Australia

Margaret Fry: Margaret.Fry@uts.edu.au

Kirsten Abrahamse: Kirsten.Abrahamse@health.nsw.gov.au

Steve Kay: stevekaywork@yahoo.com.au

Rosalind Elliott: Rosalind.Elliott@uts.edu.au

\section{Corresponding Author:}

Dr Rosalind Elliott

Clinical Nurse Consultant - translational research co-ordinator

Research and Practice Development

Northern Sydney Local Health District

Email: Rosalind.Elliott@sydney.edu.au

\section{Conflict of Interest}

The authors declare that they have no competing interests in relation to the study reported in this manuscript or the manuscript.

\section{Ethical Statement}

This study was undertaken after ethical and scientific review by the Northern Sydney Local Health District lead Human Research Ethics Committee (approval no. LNR/15/HAWKE/108)

\section{Funding Source}

None.

\section{Clinical Trials Registry and Registration number}

Not applicable.

\section{Acknowledgments}

Authors sincerely thank the emergency nurses for their participation in the survey and detailed responses and insights. 


\section{Abstract}

Introduction: Suicide in older people is a public health concern. Emergency nurses are ideally placed to identify suicide risk. Therefore, the aim of this research was to explore emergency nurses' knowledge, confidence and attitudes about suicide in older people.

Methods: This descriptive exploratory study was conducted in four emergency departments in Sydney, Australia. Data were collected using a 28-item survey from a convenience sample of emergency nurses. Descriptive quantitative statistics and conventional content analysis were performed. Ethics approval was provided.

Results: The response rate was $58 \%(n=136)$; the majority were female with an average of seven years emergency experience. The majority $(n=124,91 \%)$ reported that they frequently managed suicidal behaviour and recognized suicide as a common event $(80 \%) .51 \%(n=69)$ recognized that suicide was a common event for older people. Only $16 \%(n=22)$ reported receiving suicide prevention training with $11 \%$ feeling confident in managing suicidal behaviour.

Conclusion: The findings contribute to the discourse on how suicide in older people is recognised by emergency nurses. Few nurses considered it a problem for older people and were not confident about their knowledge. There is a need for suicide prevention training as a priority particularly to identify risks in older people.

Keywords: emergency care, aged, suicide, nurses, perception, attitudes 


\section{TITLE: SUICIDE IN OLDER PEOPLE, ATTITUDES AND KNOWLEDGE OF EMERGENCY NURSES: A MULTI-CENTRE STUDY}

\section{Introduction}

\subsection{Background and importance}

Suicide rates are increasing worldwide [1-3]. Additionally, completed suicides rates in older people (over 65 years) have also increased [4]. In Australasia there are approximately 2500 completed suicides reported annually. Significantly, people aged 85 years and older appear are three times more likely to suicide than the rest of the population [5] which is reflected in data worldwide [6]. Importantly, the number of men over 85 years who complete suicide is more than double that of male teenagers [5]. Of note suicide patterns in older people suggest that males have a higher mortality rate compared to females $[7,8]$. Older people are more likely to take lethal measures when attempting suicide, which results in fewer suicide attempts per individual [9]. Suicide in older people is a growing public health issue, which requires further understanding and preventative action [10].

Suicidal behaviour and ideation is defined as a continuum of self-risk, often recognized by the most significant negative outcome, death [10]. This may manifest in overt attempts to complete suicide or deliberate self-neglect (e.g. not taking life sustaining medication) resulting in an insidious decline in health (and thus a likely under-reporting of the true suicide rate) [11]. Older people are exposed to a plethora of risk factors associated with suicide, such as social isolation, increased incidence of depression, substance abuse, aging processes, physical co-morbidities, chronic conditions, and grief [12-15]. The complex interaction of such risk factors further compounds the risk of suicide for older people $[13,16]$.

Co-morbidities such as neurodegenerative disorders are often a devastating companion of aging [17]. Evidence suggests that people with neurodegenerative disorders, such as early onset dementia, 
are particularly vulnerable and often contemplate suicide at the time of diagnosis [18]. The risk of suicide has been found to be higher in the early stages of neurodegenerative disorders; when the individual has capacity and ability to plan suicide [19].

It is known that people are frequently in contact with a health provider thirty days prior to suicide, often presenting with somatic or non-mental health related complaints [14, 20]. However, older people tend to be less vocal about their suicidal ideation and distress prior to suicide, and common warning signs are subtle and often hidden [21]. Consequently, it can be difficult for healthcare professionals to identify suicidal behaviour or ideation in older people. While there are a number of screening tools used in Australia, little is known about the assessment and management of the suicidal older person in the emergency department (ED). The discourse surrounding suicide in older people is an important step in preventing avoidable death and may contribute to vital changes in the way older people are assessed and managed in the ED.

The ED provides an incredible opportunity for early identification of older people who are at risk of suicide. Emergency nurses are the main health providers to interact and identify patients at risk and therefore are ideally placed to improve outcomes for older people presenting with suicidal behaviour [22]. Studies indicate that primary health professionals and care of the elderly health care workers frequently do not recognize the risk of suicide in older people even when there are warning signs [23] and lack insight into the problem [24, 25]. However little is known about emergency nurses' expertise, knowledge and attitudes to older people with mental illness and suicidal behaviour. Given that this can influence the quality of assessment, documentation and management offered to this vulnerable cohort [26] there is an imperative explore emergency nurses' attitudes and knowledge. Therefore, the aim of this research was to explore emergency nurses' knowledge, confidence and attitudes about caring for older suicidal people.

\section{Methods}

This was a descriptive exploratory study using quantitative (survey) methods. 


\subsection{Site}

A multicentre study was conducted across four EDs within one local health district in metropolitan Sydney, Australia. The health district includes four public hospitals, of which one is a level 6 tertiary referral hospital and three are level 5 district hospitals [27]. The local health district provides services for people located over 900 square kilometers (population catchment: 853,000 ).

\subsection{Sample}

Convenience sampling was used. Emergency nurses employed within the four EDs were invited to participate in a survey via recruitment posters and announcements at department meetings. The inclusion criteria included: nurse managers, registered nurses and/or enrolled nurses employed full time or part time in any of the four EDs. Exclusion criteria included assistants in nursing; student nurses, casual and agency nurses.

\subsection{Survey Instrument}

A 28-item survey was developed based on expert opinion (to ensure face validity) and published research. The survey items were designed to explore knowledge, skills, confidence and attitudes towards caring for the older suicidal person presenting to the ED. Many items ( $n=12)$ were closed requiring a 'yes' or 'no' answer and others requiring a response on a 5 point likert scale $(n=8)$. Two items required free text responses; one was an opportunity to add comments to response. The reliability and validity of the survey was not formally explored however two emergency nurse clinicians (one with extensive research expertise) checked the face validity of the survey.

\subsection{Data collection and analysis}

Nurses were not approached individually by investigators; the survey (paper) was placed in staff areas for the respondents' convenience. A sealed box was located at the nurse's station and staff room for survey return at a time of the respondents' choosing. The quantitative data related to the 
survey responses were entered and analysed into an Excel document. Incomplete surveys with more than $20 \%$ of items missing were excluded from the analyses.

The study was not designed to test a hypothesis and therefore a sample size calculation was not performed. However every effort was made to invite all nurses working in the study sites in order to obtain a representative sample of nurses working in metropolitan hospitals. Simple descriptive statistics were used to describe the sample and explore the survey data that is counts and frequencies for categorical data and means (standard deviations) and medians (inter-quartile ranges) for continuous data. Conventional content analysis [28] that is counts and categories of words with similar meanings was used to explore the free text responses; the authors reviewed and agreed on the categories that emerged.

\subsection{Ethics Approval}

Ethical approval to undertake the study was obtained from the Local Health District lead Human Research Ethics Committee (approval no. approval no. LNR/15/HAWKE/108). The survey was anonymous and therefore privacy, confidentiality and beneficence were assured. Respondents were provided with a study information sheet and consent implied on completion and return of the survey.

\section{Results}

\subsection{Characteristics of the respondents}

Data were collected for three months from April to June 2016. There was a $58 \%$ response rate ( $n=136)$. The demographic characteristics of the respondents are shown in Table 1 . The majority of respondents were registered nurses ( $n=101,74 \%)$, female $(n=115,85 \%)$ with a mean age of 35.0 (11.2) years and 7.2 (7.4) years' experience of emergency nursing.

[Table 1] 


\subsection{Survey findings}

The survey findings are presented in Table 2 which demonstrates that $91 \%(n=124)$ of respondents reported that they had directly cared for someone with suicidal behaviour or ideation. Nearly half of respondents $(n=61)$ reported encountering suicidality more than 5 times per month and a large proportion had been present at the time a person presented with self-harm or suicide.

Of the respondents, $102(80 \%)$ agreed that suicide was a common event in the general population but only about half ( $n=69,51 \%$ ) of the respondents agreed that it was common in older people. A further $27(20 \%)$ respondents were unsure if it was a common event in older people. Instead, respondents considered the $18-35$ year age group as most susceptible to suicide.

Although some respondents agreed that suicide was a common event in older people experiencing mental health problems were unsure $(n=35,26 \%)$ and half $(n=71,52 \%)$ of respondents did not agree that self-harm was common in older people. The most frequent differences in needs between younger people and older people reported by respondents were the increased need for health care related to declining health, co-morbidities and a lack of social support.

Respondents ranked male gender as the highest risk factor for suicide in older people. Previous selfharm, depression and hopelessness were also highly ranked (data not shown). Nurses ranked five protective factors to prevent suicidal behaviour specific to older people as follows: ability to ask for help ( $n=38,31.4 \%)$; family support $(n=27,22.3 \%)$; sense of humour $(n=27,22.3 \%)$; personal resilience ( $n=20,16.5 \%)$; and social inclusion $(n=9,7.4 \%)$ (data not shown). Of the respondents, 93\% $(n=127)$ agreed that depression was a warning sign for suicide, however, only $73 \%(n=100)$ thought this was relevant for older people.

Respondents most frequently selected low mood, social isolation, hopelessness and poor self-care as signs and symptoms of suicidal behaviour in older people. Half of the respondents correctly identified substance misuse as a risk factor for this population but more than half did not agree or were unsure if it was linked to suicide risk. 


\section{[Table 2]}

Only $16 \%(n=22)$ of respondents reported receiving suicide prevention training with $91 \%$ indicating that they would like suicide prevention training. Self-reports of confidence in managing suicidal behavior reflected these results as only $11 \%(n=15)$ reported feeling confident or very confident. Conversely two thirds ( $n=88,64 \%$ ) of respondents reported feeling moderately to significantly confident to assess patients with suicidal ideation. Only $70 \%(n=96)$ reported asking all patients presenting with mental health concerns if they have suicidal plans or thoughts and a half $(n=69$, $50 \%$ ) reported feeling moderately to significantly confident about exploring suicidal plans with patients. The majority of respondents' $(n=108,78 \%)$ reported some distress associated with working with people who self-harm or suicide.

Respondents provided many examples of how the needs of older people (>65years) differ from younger ( $<64$ years) people. Specifically the six themes such as 'Social isolation', 'Poor function/dependence' and 'Co-morbidities' that emerged from content analysis of their responses suggested considerable insight (Table 3). Their ranking of factors to prevent suicide in older people also revealed some insight into solutions including family support and personal resilience.

[Table 3]

\section{Discussion}

The responses of this predominately female sample of emergency nurses working in hospitals in Sydney (New South Wales, Australia) provided a useful insight into the knowledge, confidence and attitudes of emergency nurses who interact with and care for older people and who are either at risk of attempting suicide, or who have attempted an act of self-harm. The results indicated that while some emergency nurses have a strong understanding of the risk of suicide in older people, there are nurses who have little awareness of the associated risks of suicide in older people. Notably, $20 \%$ of respondents were unsure if suicide was a common event in older people and $26 \%$ were unsure if suicide was a common event in older people experiencing mental health problems. 
Given the global increase in suicide in older people emergency nurses need to be vigilant when undertaking their assessment to ensure early identification of suicidal behaviour and associated risk factors in older people [10]. In the current study, emergency nurses were able to identify that depression was a warning sign for suicide. However, only $73 \%$ respondents thought this was relevant to the older person. Perhaps the respondents of this study considered depression in older people was uncommon, and therefore the association between depression and suicide in the older person was underappreciated. Another explanation for this finding might be that, depression is often under reported and detected in this group, as many symptoms of depression or low mood and affect are incorrectly attributed to "normal aging" $[29,30]$. This attitude is reflected in low suicide risk screening rates by emergency clinicians for older people presenting to the ED [31].

Older people are living longer and with this longevity there is an associated increase in people experiencing neurodegenerative disorders, social isolation and chronic conditions which are risk factors for mental illness and may increase the likelihood of attempted suicide [4, 11]. Older people have multiple risk factors for suicide and warrant specific screening and assessment that is informed by a thorough understanding of the risk factors, protective factors and subtle signs of suicidality [10, 11]. Emergency nurses are uniquely positioned to screen for suicide risk. Thus it is vital that emergency nurses have the skills to identify and understand the unique profile of suicide in older people. This is particularly important considering the low detection rates, subtle presentation of suicidality, and the high incidence of associated suicide risk factors in this cohort.

The study findings suggest that it is imperative that emergency nurses are aware that depression is a risk factor for suicide in older people. More importantly emergency nurses need to develop greater confidence and find opportunities to assess older peoples' mental health concerns. Suicide prevention training has shown to positively affect attitudes of emergency nurses caring for suicidal patients [32]. However the effect of this training on patient outcomes has not been extensively examined and requires further research. 
The majority of respondents of this study were able to correctly identify that substance misuse is a potential problem in older people [33]. However, only a small number of participants recognised that substance abuse was linked to suicidal behaviour. Current evidence suggests that there is a casual link between substance abuse and suicide [34], and that this problem is pertinent for older people [35]. The relationship between substance abuse and suicide in older people is especially concerning considering older people are often taking prescription medications which can easily be misused or abused [36]. Further, it is known that impaired prognosis and co-morbidities are associated with substance misuse in older people [37]. This reiterates the need for vigilance when performing the triage and or health assessment on admission to the ED. Arguably, when individuals present with physical injuries, which are secondary to the misuse or abuse of substances, the physical complaint may take priority for treatment and any precipitating factors may be missed. Factors such as patient load, time constraints and limitations in the documentation at triage of the presentation (that is the priority of a physical complaint) may mean that the cause is never elucidated. It is imperative to investigate whether patients presenting with any complaint that may be associated with substance abuse accidentally or intentionally misused the substance. Arguably, elucidating this vital health history is dependent on the clinician gaining the trust of the older person, using exemplary communication skills and the ability to do this in an environment which is rushed and unfamiliar for the older person.

In our study a significant number of nurses reported low confidence when managing suicidal behaviour. Additionally, some nurses reported some level of distress when reflecting on an incident when they managed a patient with suicidal behaviour, self-harm or had taken an overdose. This may in part be a function of the low number of nurses who reported that they had received formal suicide prevention training. Suicide prevention training may provide nurses with greater confidence, resilience and a more comprehensive understanding of how to better care for older people and improve their health outcomes. 
It would also be pertinent to investigate nurses' confidence and distress in relation to this aspect of caring for older people. Further exploratory research would elucidate the underlying reasons for their low confidence and strategies to enhance self-efficacy. In addition research is required to clarify the nature and reasons for the relatively high levels of distress reported by nurses in this study. Addressing this may engender resilience and empower nurses to assist older people expertly. There are some limitations associated with the study which must be acknowledged. Although the survey was designed with care including attention to current evidence (and face validity) it was not formally tested for validity and reliability. In addition arguably the complexity of caring for suicidal people in practice is not captured adequately using likert items and 'yes'/'no' questions. The findings would have been enhanced by the addition of qualitative data collection such as semi-structured interviews.

Suicide in older people is a potentially avoidable public health problem that needs to be more thoroughly and rapidly assessed. This study investigated some aspects of emergency nurses' knowledge, confidence and attitudes, however a more in-depth understanding of this issue is required. Future research should seek to investigate how emergency departments may better detect and document primary and secondary reasons for presentations by older people. It may be that many older people seen in an ED are vulnerable to suicide, or may even have the risk factors associated with suicide such as isolation and depression, and it is this cohort which would benefit from research into suicide prevention efforts. Educational opportunities that allow nurses to clarify those areas they are unsure of may be beneficial, and importantly may provide an opportunity for nurses to develop the skills in which they may translate theoretical knowledge into actual clinical practice, thus improving the healthcare outcomes of the growing population of older people. Nurses are faced with an extraordinary responsibility to assess and refer patients for suitable interventions. Future research must consider investigating educational opportunities and their impact on nursing confidence and resilience within the domain of mental health for older people. 


\section{Conclusions}

The findings of this survey further contribute to the discourse on how depression and suicide in older people is perceived and addressed by emergency nurses. Despite frequent exposure to people presenting with suicidal behaviour relatively few nurses perceive it as a problem for older people. Indeed, many nurses were not confident about their knowledge and ability to address suicide and associated risk factors in older people. This may in part be a result of an unmet educational need that is suicide prevention training. There is an imperative to address this aspect of emergency care for older people to prevent unnecessary suffering and ensure that the population ages healthily. 


\section{References}

1. Frénisy M-C, Plassard C. Suicide in the elderly. Soins: la Revue de Reference Infirmiere. 2017,62:39-41.

2. Bonnewyn A, Shah A, Demyttenaere K. Suicidality and suicide in older people. Reviews in Clinical Gerontology. 2009,19:271-94.

3. Worldwide Suicide [database on the Internet]. 2014 [cited 31 March 2014]. Available from: http://www.who.int/en/.

4. Rhonda Nay SG, Deirdre Fetherstonhaugh Older People. Issues and Innovations in care 4th ed. Chatswood: Elsevier; 2014.

5. $\quad$ ABS. Suicide In: Statistics ABo, editor. Australia2014.

6. Bachmann S. Epidemiology of Suicide and the Psychiatric Perspective. International journal of environmental research and public health. 2018,15. Epub 2018/07/11.

7. Almeida OP, Draper B, Snowdon J, Lautenschlager NT, Pirkis J, Byrne G, Sim M, Stocks N, Flicker L, Pfaff JJ. Factors associated with suicidal thoughts in a large community study of older adults. The British Journal of Psychiatry. 2012,201:466-72.

8. Shah A, Bhat R, Zarate-Escudero S, DeLeo D, Erlangsen A. Suicide rates in five-year agebands after the age of 60 years: the international landscape. Aging \& Mental Health. 2016,20:131-8.

9. Poorolajal J, Rostami M, Mahjub H, Esmailnasab N. Completed suicide and associated risk factors: a six-year population based survey. Archives of Iranian medicine. 2015,18:39-43. Epub 2015/01/06.

10. O'Connell H, Ai vryn C, Cunningham C, Lawlor B. Recent Developments: Suicide in older People. BMJ. 2004,329:895-9.

11. Alphs L, Brashear HR, Chappell P, Conwell Y, Dubrava S, Khin NA, Kozauer N, Hartley DM, Miller DS, Schindler RJ, Siemers ER, Stewart M, Yaffe K. Considerations for the assessment of suicidal ideation and behavior in older adults with cognitive decline and dementia.

Alzheimer's \& Dementia: Translational Research \& Clinical Interventions. 2016,2:48-59.

12. Van Orden K, Conwell Y. Issues in Research on Aging and Suicide. Aging Ment Health. 2017,20:240-51.

13. Fässberg MM, van Orden KA, Duberstein P, Erlangsen A, Lapierre S, Bodner E, Canetto SS, De Leo D, Szanto K, Waern M. A Systematic Review of Social Factors and Suicidal Behavior in Older Adulthood. International journal of environmental research and public health. 2012,9:72245.

14. Turecki G, Brent D. Suicide and suicidal behaviour. Lancet. 2016:Published online 2015 Sep 15. doi: 10.1016/S0140-6736(15)00234-2.

15. Fassberg MM, Cheung G, Canetto SS, Erlangsen A, Lapierre S, Lindner R, Draper B, Gallo $\mathrm{JJ}$, Wong $\mathrm{C}$, Wu J, Duberstein $\mathrm{P}$, Waern M. A systematic review of physical illness, functional disability, and suicidal behaviour among older adults. Aging Ment Health. 2016,20:166-94. Epub 2015/09/19.

16. Draper B, Kõlves K, De Leo D, Snowdon J. A Controlled Study of Suicide in Middle-Aged and Older People: Personality Traits, Age, and Psychiatric Disorders. Suicide and Life-

Threatening Behavior. 2014,44:130-8.

17. van der Flier WM, Scheltens P. Epidemiology and risk factors of dementia. Journal of Neurology, Neurosurgery \&amp; Psychiatry. 2005,76:v2-v7. 
18. Eliasen A, Dalhoff KP, Horwitz H. Neurological diseases and risk of suicide attempt: a case-control study. Journal of neurology. 2018,265:1303-9. Epub 2018/03/23.

19. Haw C, Harwood D, Hawton K. Dementia and suicidal behavior: A review of the literature. International Psychogeriatrics. 2009,21:440-53.

20. Carter MW, Reymann MR. ED use by older adults attempting suicide. The American journal of emergency medicine. 2014,32:535-40.

21. Manthorpe J. Suicide among older people Nursing Older People. 2006,17:25-9.

22. Plawecki L. Someone to talk to The nurse and the depressed or suicidal older patient. 2010.

23. Kjolseth I, Ekeberg O. When elderly people give warning of suicide. Int Psychogeriatr. 2012,24:1393-401. Epub 2012/03/28.

24. Butler MP, Quayle E. Training primary care nurses in late-life depression: knowledge, attitude and practice changes. International journal of older people nursing. 2007,2:25-35. Epub 2007/03/01.

25. Huh JT, Weaver CM, Martin JL, Caskey NH, O'Riley A, Kramer BJ. Effects of a late-life suicide risk--assessment training on multidisciplinary healthcare providers. $J$ Am Geriatr Soc. 2012,60:775-80. Epub 2012/02/01.

26. Seidlitz L, Duberstein, PR, Cox, C, Conwell, Y. . Attitudes of older people toward suicide and assisted suicide: An analysis of Gallup Poll Findings American Geriatrics Society 1995,43:993-7.

27. Commonwealth of Australia. My Hospitals. National Health Performance Authority; 2013 [12/04/2013]; Available from: http://www.myhospitals.gov.au/.

28. Hsieh H-F, Shannon SE. Three Approaches to Qualitative Content Analysis. Qualitative Health Research. 2005,15:1277-88.

29. Murray J, Banerjee S, Byng R, Tylee A, Bhugra D, Macdonald A. Primary care professionals' perceptions of depression in older people: a qualitative study. Social science \& medicine (1982). 2006,63:1363-73. Epub 2006/05/16.

30. Ruppel SE, Jenkins WJ, Griffin JL, Kizer JB. Are they depressed or just old? A study of perceptions about the elderly suffering from depression. North American Journal of Psychology. 2010,12:31.

31. Betz ME, Arias SA, Segal DL, Miller I, Camargo CA, Boudreaux ED. Screening for Suicidal Thoughts and Behaviors in Older Adults in the Emergency Department. Journal of the American Geriatrics Society. 2016,64:e72-e7.

32. Kishi Y, Otsuka K, Akiyama K, Yamada T, Sakamoto Y, Yanagisawa Y, Morimura H, Kawanishi C, Higashioka H, Miyake Y, Thurber S. Effects of a Training Workshop on Suicide Prevention Among Emergency Room Nurses. Crisis. 2014,35:357-61.

33. Gleason LJ, Escue ED, Hogan TM. Older Adult Emergency Department Pain Management Strategies. Clinics in Geriatric Medicine. 2018,34:491-504.

34. Conner KR, Gamble SA, Bagge CL, He H, Swogger MT, Watts A, Houston RJ. Substanceinduced depression and independent depression in proximal risk for suicidal behavior. Journal of studies on alcohol and drugs. 2014,75:567-72. Epub 2014/07/06.

35. Morin J, Wiktorsson S, Marlow T, Olesen PJ, Skoog I, Waern M. Alcohol use disorder in elderly suicide attempters: a comparison study. The American journal of geriatric psychiatry : official journal of the American Association for Geriatric Psychiatry. 2013,21:196-203. Epub 2013/01/25. 
36. Koo YW, Kõlves K, de Leo D. Profiles by suicide methods: an analysis of older adults. Aging \& Mental Health. 2017:1-7.

37. Han BH, Moore AA. Prevention and Screening of Unhealthy Substance Use by Older Adults. Clin Geriatr Med. 2018,34:117-29. Epub 2017/11/14. 
Suicide in older people, attitudes and knowledge of emergency nurses: a multi-centre study Highlights

- More than $90 \%$ of emergency nurses had cared for someone with suicidal behaviour

- Half of the emergency nurses agreed that suicide was common in older people

- The 18-35 year age group was considered by nurses as most susceptible to suicide

- Male gender was ranked as the highest risk factor for suicide in older people

- $16 \%$ reported receiving suicide prevention training; $91 \%$ wanted it 
Table 1. Demographic characteristics of the sample $\left(n=136^{*}\right)$

\begin{tabular}{lc}
\hline Characteristics & Statistic \\
\hline
\end{tabular}

Female gender, $\mathrm{n}(\%)$

$115(85)$

Age, years, mean (SD)

$35.0(11.2)$

Nursing experience, years, mean (SD)

$11.7(11.7)$

ED experience, years, mean (SD)

$7.2(7.4)$

Employment role, $\mathrm{n}(\%)$

Registered Nurse

$101(74)$

Clinical Nurse Specialist

$10(8)$

Nurse Unit Manager

$9(7)$

Clinical Nurse Educator

$7(5)$

Clinical Nurse Consultant/ Clinical Nurse Specialist grade 2

$5(3)$

Enrolled Nurse

$4(3)$

Qualifications, n (\%)

Bachelor

$77(56)$

Hospital certificate

$13(9)$

Masters

$12(8)$ 
Other

Employment status, n (\%)

Full time

Part time

Casual employees

Type of facility, n (\%)

Tertiary referral hospital

Metropolitan hospital
$96(70)$

$36(26)$

$2(1)$

$28(21)$

$105(77)$

*up to 3 missing data for some items; SD = standard deviation 
Table 2. Results of closed question survey responses $(n=136)$

\begin{tabular}{|c|c|c|c|c|c|c|c|c|c|}
\hline \multirow[t]{2}{*}{ Item no. } & \multicolumn{3}{|l|}{ Content } & \multicolumn{6}{|l|}{$\mathrm{n} *(\%)$} \\
\hline & & & & \multicolumn{2}{|l|}{ Yes } & \multicolumn{2}{|l|}{ No } & \multicolumn{2}{|c|}{ Unsure } \\
\hline 1. & Suicide common event & Australia & & $109(80$ & & $8(6)$ & & $15(11)$ & \\
\hline \multirow[t]{2}{*}{2.} & \multicolumn{3}{|c|}{ Suicide common event in older people } & \multicolumn{2}{|l|}{$69(51)$} & \multicolumn{2}{|l|}{$37(27)$} & \multicolumn{2}{|l|}{$27(20)$} \\
\hline & Age, years & $18-25$ & $25-35$ & $35-45$ & $45-55$ & $55-65$ & $65-75$ & $75-85$ & $85+$ \\
\hline \multirow[t]{3}{*}{3.} & Age group most & 52 & 33 & 15 & 7 & 5 & 3 & 6 & 4 \\
\hline & susceptible to suicide & (38) & (24) & (11) & (5) & (3) & (2) & (4) & (3) \\
\hline & for males & & & & & & & & \\
\hline \multirow[t]{3}{*}{4.} & Age group most & 54 & 39 & 11 & 14 & 1 & 3 & 4 & 3 \\
\hline & susceptible to suicide & $(40)$ & (29) & (8) & (10) & - & (2) & (3) & (2) \\
\hline & & & & \multicolumn{2}{|l|}{ Yes } & \multicolumn{2}{|l|}{ No } & \multicolumn{2}{|c|}{ Unsure } \\
\hline 5 & \multicolumn{3}{|c|}{ Suicide more common event in older } & \multicolumn{2}{|l|}{$79(58)$} & \multicolumn{2}{|l|}{$19(14)$} & \multicolumn{2}{|c|}{$35(26)$} \\
\hline 6 & Self-harm common in $\mathrm{c}$ & er peopl & & $30(22)$ & & $71(52)$ & & $31(23)$ & \\
\hline 8. & Depression a warning s & for suic & & $127(93$ & & $4(3)$ & & $2(1)$ & \\
\hline 9. & Depression a more con & Ion warn & g sign & $100(73$ & & $9(6)$ & & 19 (14) & \\
\hline
\end{tabular}


10. Substance misuse a problem in older

$$
\text { person }
$$

11. Suicide linked to substance abuse in older$$
\text { person }
$$

12

Which substances commonly misused by

older person, $\mathrm{n}$

Alcohol: 99

Benzodiazepines: 84

Nicotine: 33

Caffeine: 12

Cannabis: 2

Stimulants (such as Amphetamine): 2

13. Common signs and symptoms of suicidal behaviour in older people
Low mood: 118

Hopelessness: 97

Insomnia: 85

Planning suicide: 73

Thoughts/plans to harm

self: 68
Social isolation: 114

Poor self-care: 96

Apathy: 77

Finalisation

personal matters:

70

Self harm: 59

Page 4 of 8 
Agitation: 52

Yes

$124(91)$

$7(5)$

behaviour and or ideation

15. Had suicide prevention training

16. Like suicide prevention training

17. How confident managing suicidal

behaviour

18. How often per month encounter suidality

behaviour

Never Once $>3 \quad>5 \quad$ Rarely

$\begin{array}{lll}\text { Not at Little Mod A lot } & \text { Very } \\ \text { all } & & \text { often }\end{array}$

$6(4) \quad 35(26) \quad 75(55) \quad 12(9) \quad 3(2)$

Yes No

19. Working when a person suicided or self-

$105(77)$

26 (19)

harmed

Not at A little Mod A lot Sign
all


20. Gauge your distress experienced looking $3(2) \quad 40(29) \quad 50(37) \quad 16(12) \quad 7(5)$ after people who suicide/self-harm

21. Challenging to care for mental health $\quad 3(2) \quad 14(10) \quad 37(27) \quad 44(32) \quad 34(25)$ patients

22. Difficult to care for someone deliberate $4(3) \quad 11(8) \quad 54(40) \quad 48(35) \quad 15(11)$ self-harm

23. Level of distress today suicidal, self-harm, $17(12) \quad 45(33) \quad 44(32) \quad 13(9) \quad 6(4)$ overdose patients

24. Confident feel assessing patients suicidal $3(2) \quad 41(30) \quad 58(43) \quad 27(19) \quad 3(2)$ ideation

25. Ask about suicidal plans or thoughts

\begin{tabular}{lll} 
Yes & No \\
\hline $96(70)$ & $32(23)$ & \\
Not at A little Mod & A lot Sign \\
all & &
\end{tabular}

26. Confidence exploring suicidal plans with $11(8) \quad 51(37) \quad 41(30) \quad 21(15) \quad 7(5)$ patients

*Up to 3 data items missing for some participants; not all percentage values add up to $100 \%$ 
Table 3. Results of free text response to survey item 7 ('How do older people's needs differ to that of the younger adult population ( $<64$ years)?', $n=136)$

Categories Exemplars

Co-morbidities/poor

'Their past medial history may play a role' (participant 21)

health/chronic health

'Failing health' (participant 76)

'More co-morbidities' (participant 130)

Social isolation

'Less social network' (participant 35)

'Loneliness' (participant 74)

'Social isolation harder to meet people' (participant 137)

Poor function/dependence

'Require more assistance with ADLs' (participant 19)

'Loss of independence' (participant 28)

'Decrease in independence - need more support'

(participant 55)

Cognitive impairment

'Decline in cognitive functions' (participant 60)

'Dementia' (participant 69)

'Onset of dementia/Alzheimer's can affect needs'

(participant 126)

Financial concerns

'Financial needs' (participant 36)

'Medication and finance' (participant 91)

'loss of work and financial independence' (participant 136) 
High risk suicide / depression

'increase incidents and severity of depression' (participant

20)

'Suicide in elderly may be more methodical and planned'

(participant 112)

'vulnerable to depression and suicide' (participant 119) 